\title{
Indoor versus outdoor scene recognition for navigation of a micro aerial vehicle using spatial color gist wavelet descriptors
}

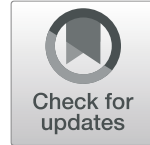

\author{
Anitha Ganesan and Anbarasu Balasubramanian * (D)
}

\begin{abstract}
In the context of improved navigation for micro aerial vehicles, a new scene recognition visual descriptor, called spatial color gist wavelet descriptor (SCGWD), is proposed. SCGWD was developed by combining proposed Ohta color-GIST wavelet descriptors with census transform histogram (CENTRIST) spatial pyramid representation descriptors for categorizing indoor versus outdoor scenes. A binary and multiclass support vector machine (SVM) classifier with linear and non-linear kernels was used to classify indoor versus outdoor scenes and indoor scenes, respectively. In this paper, we have also discussed the feature extraction methodology of several, state-of-the-art visual descriptors, and four proposed visual descriptors (Ohta color-GIST descriptors, Ohta color-GIST wavelet descriptors, enhanced Ohta color histogram descriptors, and SCGWDs), in terms of experimental perspectives. The proposed enhanced Ohta color histogram descriptors, Ohta color-GIST descriptors, Ohta color-GIST wavelet descriptors, SCGWD, and state-of-the-art visual descriptors were evaluated, using the Indian Institute of Technology Madras Scene Classification Image Database two, an Indoor-Outdoor Dataset, and the Massachusetts Institute of Technology indoor scene classification dataset [(MIT)-67]. Experimental results showed that the indoor versus outdoor scene recognition algorithm, employing SVM with SCGWDs, produced the highest classification rates (CRs) - 95.48\% and 99.82\% using radial basis function kernel (RBF) kernel and 95.29\% and 99.45\% using linear kernel for the IITM SCID2 and Indoor-Outdoor datasets, respectively. The lowest CRs_-2.08\% and 4.92\%, respectivelywere obtained when RBF and linear kernels were used with the MIT-67 dataset. In addition, higher CRs, precision, recall, and area under the receiver operating characteristic curve values were obtained for the proposed SCGWDs, in comparison with state-of-the-art visual descriptors.
\end{abstract}

Keywords: Micro aerial vehicle, Scene recognition, Navigation, Visual descriptors, Support vector machine

\section{Introduction}

Classification of a scene as being indoors or outdoors is a challenging task in the navigation of a micro aerial vehicle (MAV). Outdoor scenes, due to different weather conditions and the wide variety of objects involved, as well as their unstructured nature, are much harder to recognize than indoor scenes. Better indoor versus outdoor scene categorization will help MAV navigation where global positioning systems (GPS) signals are not available. Blockage or interruption of GPS signals by dense and tall buildings, by trees and inside buildings occurs in both the indoor and outdoor environment,

\footnotetext{
* Correspondence: avianbu@gmail.com

Department of Aerospace Engineering, Madras Institute of Technology, Anna University, Chennai 600 044, India
}

Springer Open

(c) The Author(s). 2019 Open Access This article is distributed under the terms of the Creative Commons Attribution 4.0 International License (http://creativecommons.org/licenses/by/4.0/), which permits unrestricted use, distribution, and reproduction in any medium, provided you give appropriate credit to the original author(s) and the source, provide a link to the Creative Commons license, and indicate if changes were made.

and to overcome this limitation, the MAV (Fig. 1) needs to have the ability to recognize the difference. A scene classification method has been proposed for indoor versus outdoor scene classification, using a probabilistic neural network that extracts color features, texture features and shape features [1] from the indoor and outdoor images.

Others have considered this issue. Payne and Singh [2] proposed a method based on edge straightness information, extracted from indoor and outdoor images, for efficient indoor and outdoor image classification. A circular thresholding method [3], based on Otsu's algorithm, used color features, texture features and Otsu features, for indoor versus outdoor image classification. Semantic, high-level, scene image properties [4] can be identified 


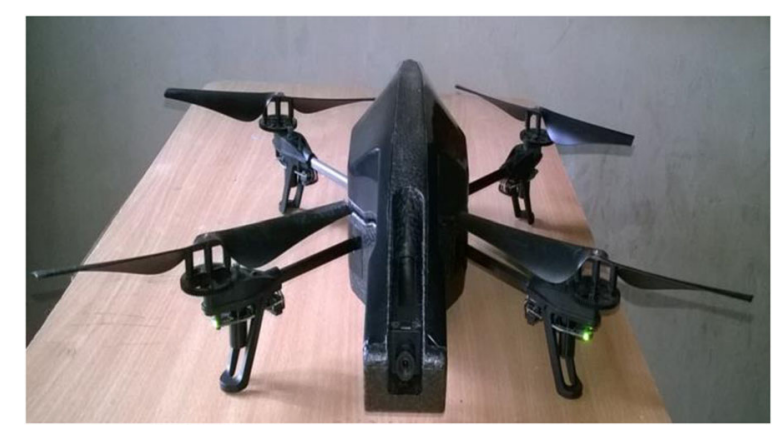

Fig. 1 Parrot augmented reality drone2 quadrotor

in low level features extracted from the sub blocks of an image, and Quattoni and Torralba [5] used local and global spatial properties of a scene for indoor scene recognition. Videos of indoor environments (corridors, staircases, and rooms), captured by the forward-facing camera of the MAV (Fig. 1) were transmitted, with an image resolution of $1280 \times 720$ pixels, via a Wi-Fi ad-hoc connection between an augmented reality (AR) drone and a ground station. Another indoor scene classification method [6] has been proposed that combines orientational pyramid matching features with spatial pyramid matching (SPM) features, using 3 dimensional orientational features to discriminate between confusing indoor scene categories.

Nearest neighbor-based metric functions [7] and Bag-ofvisual word schemes have been used to recognize indoor scenes, while local image regions have been represented by contextual visual words [8] for scene categorization. In another study, a Scene Understanding (SUN) database [9] was proposed for large-scale scene recognition, using 130,519 images from 899 scene categories.

Efficient scene recognition methods [10-12] have been proposed by researchers across the world. Recently, region-based contextual visual information, integrated with the Bag-of-visual words approach and contextual visual word-based image representation [13] of the scene, was proposed for efficient scene categorization. In another study, a query binary image retrieval method [14] was proposed, using deep belief networks and a Softmax classifier, while neural network classifiers [15] have been used as a tool to categorize remotely sensed images.

A divisive information theoretic feature clustering algorithm [16] has been used to create compact, pyramidbased image representation for object and scene recognition, while a sparse coding-based, SPM kernel [17] was proposed to capture salient image properties for use in image categorization, by applying sparse coding and spatial max pooling to scale-invariant feature transform (SIFT) descriptors. Spatial pyramid image representation, achieved by computing histograms of SIFT descriptors
[18] extracted from image sub-regions, has been proposed. In a separate study, several state-of-the-art visual descriptors [19] were evaluated, using classification accuracy ratings for four benchmark scene data sets, such as an eight-outdoor-scene data set, a 15-scene data set, a 67-indoor-scene data set, and the SUN397 data set. The major contribution of this study was the integration of proposed Ohta Color-GIST wavelet descriptors and CENTRIST (spatial pyramid representation) descriptors, to recognize complex indoor versus outdoor scenes for MAV navigation in GPS-denied indoor and outdoor environments. Finally, several additional scene recognition methods [20-22] have been proposed, for indoor versus outdoor scene and indoor scene categorization.

\section{Related scene recognition visual descriptors}

Visual descriptors-such as SIFT-ScSPM, SIFT-LLC, SIFT-SPM, histogram of oriented gradients (HOG)SPM, Enhanced-GIST, CENTRIST, CENTRIST (spatial pyramid representation), speeded-up Robust Features (SURF)-SPM, Color-GIST descriptors, Ohta Color-GIST wavelet descriptors, and spatial color gist wavelet descriptors (proposed visual descriptors) - have been used for indoor versus outdoor scene categorization. Several of the feature extraction methods used in these descriptors for scene categorization have been discussed in the following sub-sections.

\section{Sift}

SIFT keypoints extracted using the SIFT algorithm proposed by Lowe [23] are immune to rotation, translation, and image illumination and scaling changes. In this method, scalespace extreme detection, keypoint localization, orientation assignment, and calculation of keypoint descriptors are the four steps involved in SIFT descriptor extraction. Difference Gaussian filters are applied to the image frames to identify stable keypoint locations in scale space, in a step where, to detect stable keypoints, unstable keypoints below the threshold value are discarded. For each stable keypoint, orientations are assigned by computing the local image gradient, before gradient magnitudes and orientations are computed for each keypoint, to extract any SIFT descriptor in the $16 \times$ 16 neighborhood of the pixel. Gradient magnitude and orientation are weighted using a Gaussian window around the location of the keypoint, to create orientation histograms with 8 bins over $4 \times 4$ (16 regions) sub regions, so that a 128-element, feature vector SIFT descriptor is extracted from each $4 \times 4$ sub-region. SIFT key points extracted from an indoor image are shown in Fig. 2a, in which the circle center and radius represent the detected SIFT keypoints and the average keypoint scale, respectively. Average keypoint orientation is represented by the arrow inside the circle. 


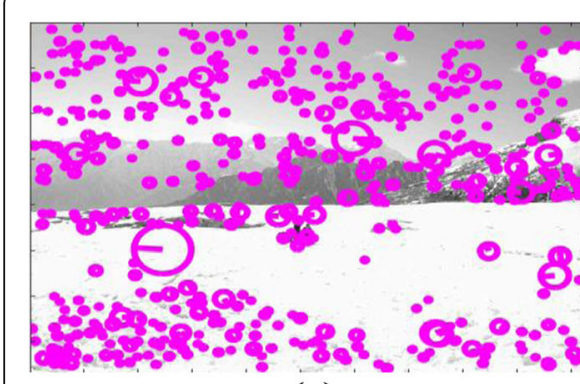

(a)

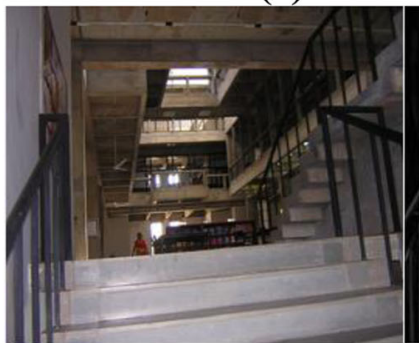

(d)

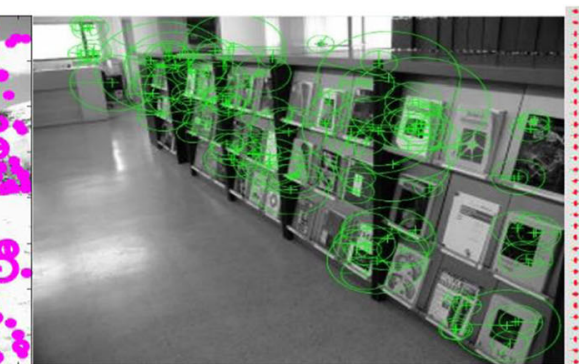

(b)

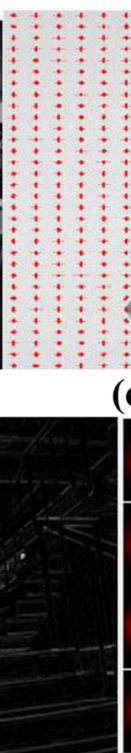

(f)

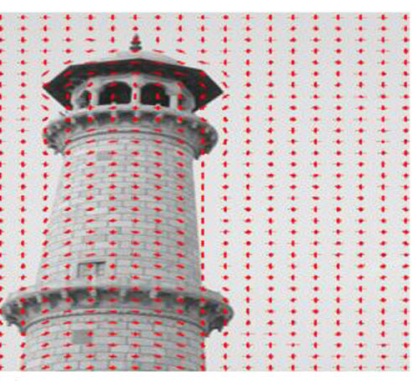

(c)

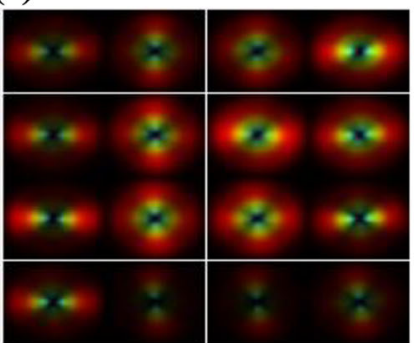

(g)

Fig. 2 Illustration of visual descriptors. a Scale-invariant feature transform key points detected in an outdoor image; b Speeded up robust features key points detected in an indoor image; $\mathbf{c}$ Histogram of oriented gradients features detected in an outdoor image; $\mathbf{d}$ Input indoor image; e Horizontal directional morphological gradient; f Vertical directional morphological gradient; $\mathbf{g}$ GIST descriptor

\section{Surf}

As for the SIFT descriptor, a scale invariant and rotation invariant visual descriptor [24] has been proposed, using determination of a hessian matrix. In this method, keypoints are selected from the detected SURF interest points in multi-scale space, using non-maximum suppression. Next, within a sliding orientation window, the sum of all Haar wavelet responses is calculated, to estimate the orientation of the keypoint with the longest vector. The Haar wavelet responses for each $4 \times 4$ subregion around the key point are computed, in the horizontal $\left(d_{x}\right)$ and the vertical $\left(d_{y}\right)$ directions, so that, for each $4 \times 4$ sub-region, the feature vector is denoted as $v=\left(\sum d_{x}, \sum d_{y}, \Sigma\left|d_{x}\right|, \Sigma\left|d_{y}\right|\right)$. SURF key points are extracted as shown in Fig. $2 \mathrm{~b}$. The circle center and radius represent the detected SURF keypoints and the average keypoint scales, respectively. Average keypoint orientation is represented by the arrow inside the circle.

\section{Hog}

The HOG descriptor [25] was proposed to detect pedestrians in grayscale images. In the HOG method, local gradient orientation histograms are computed from grayscale images, to extract the HOG descriptor. Firstly, image gradients along the horizontal and vertical directions are computed, followed by dividing the image into circular or rectangular connected regions-each with the dimensions of $16 \times 16$ pixels-called cells. Thirdly, gradient orientation histograms of are computed within each cell's pixels, and each cell's pixels contribute to the weighted score for a histogram. Finally, the L2-norm method is used to normalize cell histograms ( 9 bins) to obtain the HOG descriptor. A HOG descriptor detected in a grayscale image for $16 \times 16$ pixel cell size can be seen in Fig. 2c.

\section{Centrist}

CENTRIST is a scene categorization visual descriptor developed [26] by replacing each pixel intensity value in a grayscale image with a census transform $(\mathrm{CT})$ value. In this method, to convert the input grayscale image into a Census Transformed image (Fig. 3b) the center pixel intensity is compared with those of its $3 \times 3$-pixel neighborhood. Bit " 1 " is assigned to the neighboring pixel if the center pixel value is greater than the value of the neighboring pixel; otherwise the pixel value is set to "0". After obtaining the CT value (Fig. 3c), histograms of CT values are used to obtain the 256-dimensional CENTRIST descriptor (not using principal component analysis). To extract CENTRIST descriptor spatial representation, an indoor or outdoor image is divided into 31 blocks $(25+5+1)$, for a level 2,1 , and 0 split in a spatial pyramid. The CENTRIST descriptors extracted from the 31 image blocks are then concatenated, to produce a spatial representation of the $7936(31 \times 256)$ dimensional CENTRIST descriptor. Therefore, to reiterate, CENTRIST descriptors with spatial pyramid representation and CENTRIST descriptors differ from each other in the feature extraction stage. 


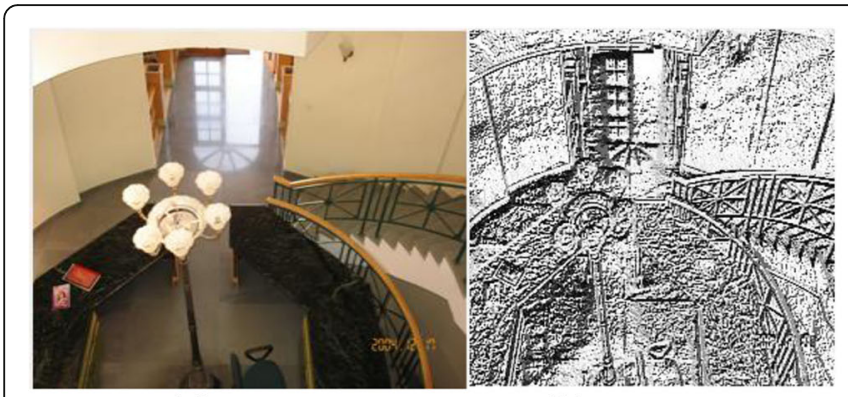

(a)

(b)

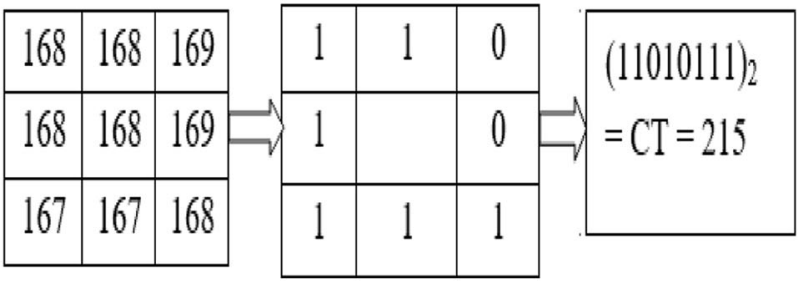

(c)

Fig. 3 Census transformed output. a Indoor image; b Census transformed Indoor image; c Census transformed value

\section{Enhanced-GIST descriptor}

The Enhanced-GIST descriptor method was proposed [27] to recognize corridors, staircases, and room types, in indoor scenes, by encoding the spatial envelope and geometric structure of the indoor scene. In this method, a bank of 32 Gabor filters is applied to an indoor and outdoor grayscale image $(256 \times 256$ pixels $)$, at 4 scales and 8 orientations, to produce 32 feature maps. These 32 feature maps are then divided into $4 \times 4$ grids, and the filtered outputs within each of the 16 regions are averaged, to produce a 512-dimensional $(16 \times 32)$ GIST descriptor (Fig. 2g). Directional morphological gradient [28] features (Fig. 2e and f) are extracted for horizontal and vertical direction $\alpha$, by using a line structuring element, as shown in Eq. (1):

$$
g L_{\alpha}(f)=\delta L_{\alpha}(f)-\varepsilon L_{\alpha}(f)
$$

where $\delta L_{\alpha}(f)$ denotes the dilated image and $\varepsilon L_{\alpha}(f)$ denotes the eroded image. Histograms of horizontal and vertical directional morphological gradient (HODMG) features are used for scene classification. GIST (512-dimensional) and HODMG (512-dimensional) descriptors are then combined, to produce 1024-dimensional Enhanced-GIST descriptors.

\section{Bag-of-words algorithms}

Bag-of-words algorithms-such as SIFT-SPM, SIFTScSPM, and SIFT-LLC-have been employed for indoor versus outdoor scene classification. In the feature extraction phase, dense SIFT features are extracted from a regular grid, and quantized into discrete visual words, and the SPM algorithm was proposed [18] to categorize natural scenes. To extract SIFT-SPM, HOG-SPM, and SURF-SPM from indoor and outdoor images, the input image is first divided into regular grids (grid spacing of 8 pixels), and SIFT, SURF and HOG descriptors are extracted from each grid. The visual vocabulary is then formed, by applying k-means clustering to the extracted features. The vector quantization (VQ) technique is then applied, to form discrete visual words, with local features represented based on the trained dictionary (codebook). Spatial histograms of coded features constitute the final feature vectors. In the sparse coding-based, SPM method proposed by Yang et al. [17], the VQ method was replaced with the sparse coding method, to quantize the local features used in SPM. In SPM and ScSPM, the histograms and the max operator are used for spatial pooling, and locality-constrained linear coding (LLC) [29] is used for image classification. Linear-weighted combinations of similar bases are learned from the trained visual words, and represent local features.

\section{Wavelet descriptors}

In this method, an input RGB image is converted into CIELAB (Lab) color space, and the resultant Lab color space image is re-sized with a scale factor value of $1 / 16$. Wavelet descriptors [30] are computed by means of three-level wavelet decomposition, using a Biorthogonal wavelet transform. The Lab color space and 2-D wavelet decomposition image are shown in Fig. 4. The 2-D (2 dimensional) wavelet decomposition is performed on the " $\mathrm{L}$ " channel of the Lab color space, and the approximation coefficients at level 3 are extracted using biorthogonal wavelets (Bior 2.6).

Laplacian filtering is then applied to the extracted approximation coefficients, to obtain the Laplacianfiltered coefficients. Finally, the percentage of wavelet energy for 2-D wavelet decomposition-corresponding to the approximation coefficients (Laplacian-filtered coefficients), and the horizontal, vertical, and diagonal detail coefficients-is computed, and is used as a feature vector for indoor and outdoor image classification.

\section{Color-GIST descriptors}

Visual perception of a scene from red, green, and blue channel, RGB indoor and outdoor images, at a glance, is known as Color-GIST recognition of an indoor versus outdoor scene. Red channel, blue channel and green channel information is extracted from the input RGB image (resolution: $256 \times 256$ pixels). For the red channel, blue 

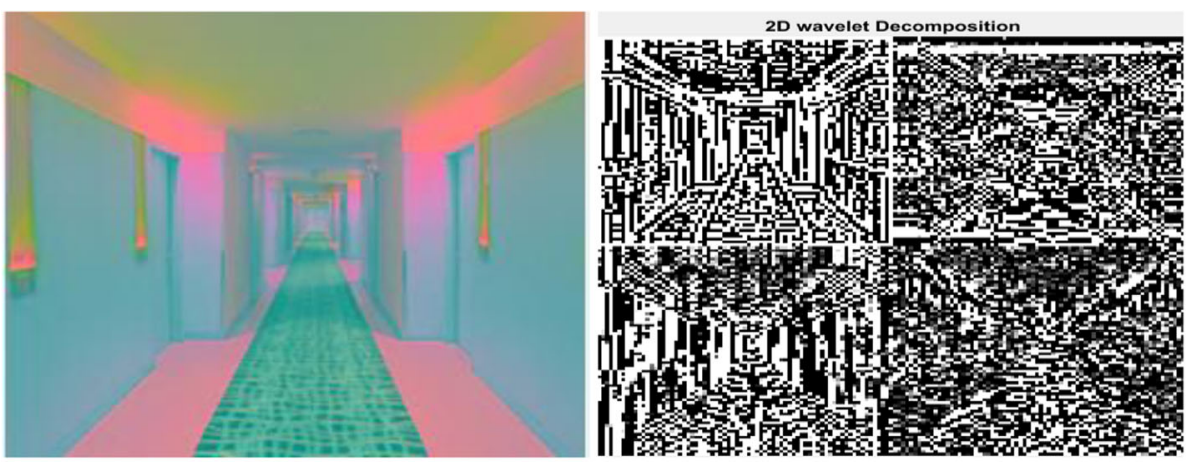

(a)

(b)

Fig. 4 Wavelet descriptors. a CIELAB color space image; b 2 dimensional wavelet decomposition output

channel, and green channel, GIST descriptors [31] are extracted by applying 64 Gabor filters, at 4 scales and 16 orientations, to produce 64 feature maps. In each channel, the resultant maps are divided into 16 regions, and the filtered outputs within each region are then averaged to produce 1024-dimensional $(64 \times 16)$ descriptors. Color-GIST descriptors (Fig. 5) are extracted by concatenating the GIST descriptors extracted from each channel, to produce a 3072-dimensional $(1024 \times 3)$ descriptor.

\section{Methods}

Real time indoor and outdoor scene recognition capabilities are needed, in order to navigate an MAV successfully. By recognizing scenes as indoor or outdoor, an MAV can follow a navigation strategy suitable for the performance of high level missions. A block diagram for a proposed indoor versus outdoor scene recognition framework is shown in Fig. 6.

The indoor versus outdoor scene recognition model consists of training and testing stages. In the training stage, visual descriptors, such as Ohta Color-GIST wavelet descriptors and CENTRIST (spatial pyramid) descriptors, are extracted from indoor and outdoor training images. The extracted SCGWDs are learned using a support vector machine (SVM) classifier, with linear and nonlinear kernels. In the testing stage, SCGWDs are extracted from the test image, and the SVM classifier with linear and nonlinear kernel classify the scene category as indoor or outdoor based on the scene recognition model learned at the training stage.

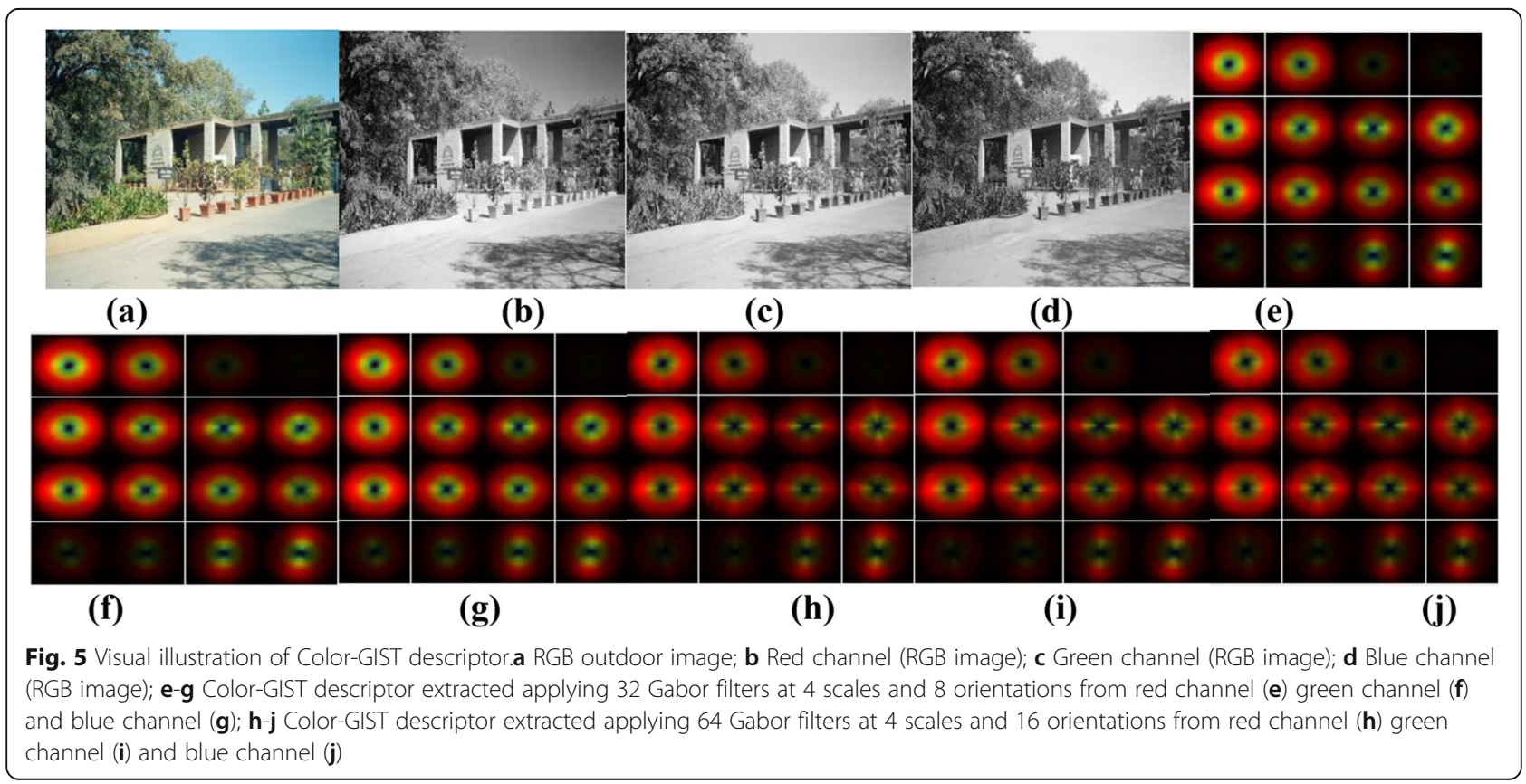




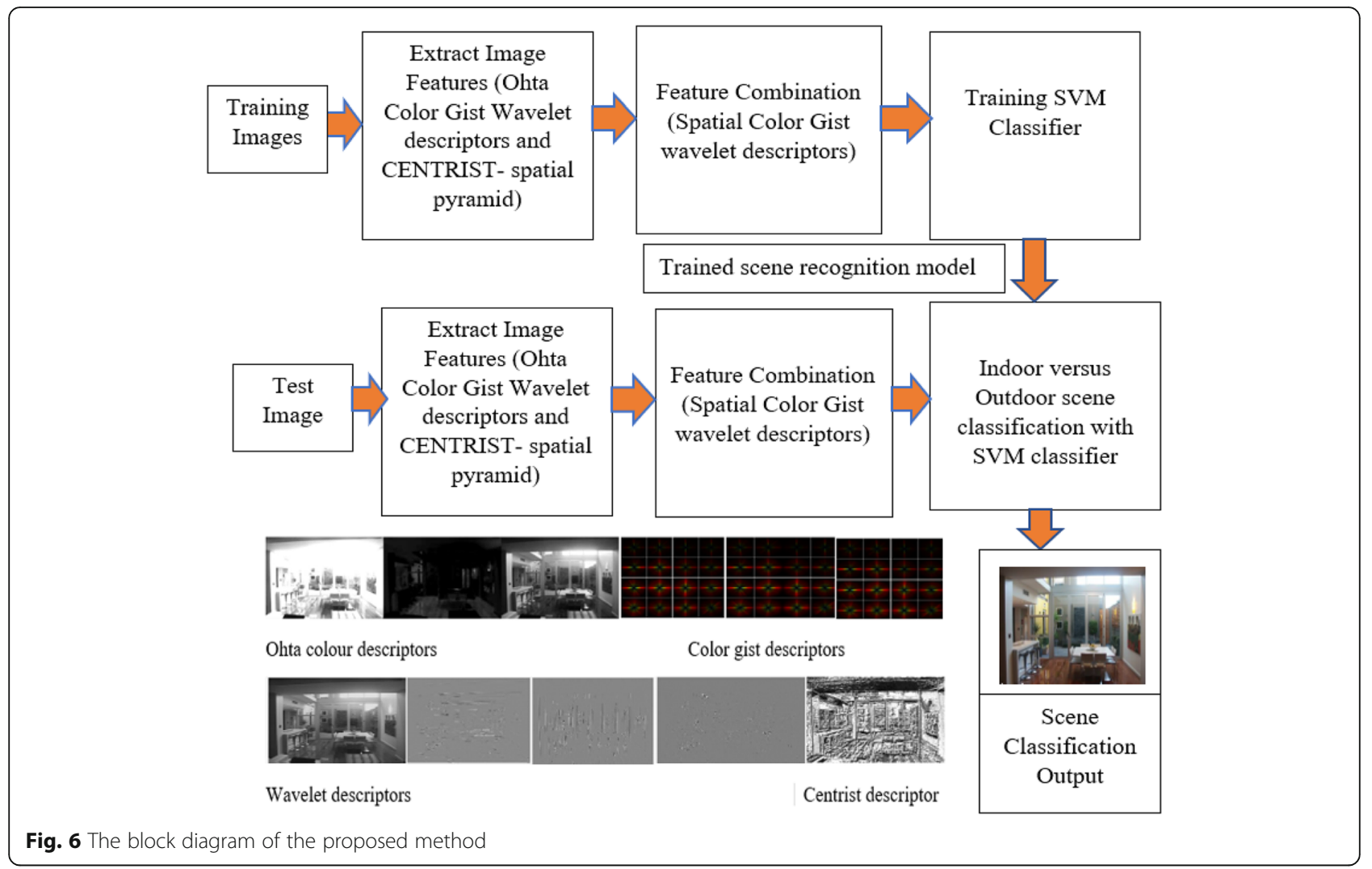

This study included comparative testing between several state-of-the-art visual descriptors and the proposed SCGWD, from both experimental and methodological perspectives, for indoor versus outdoor scene categorization tasks performed in the challenging Indian Institute of Technology Madras Scene Classification Image Database two (IITM-SCID2), an Indoor-Outdoor Dataset, and the Massachusetts Institute of Technology indoor scene classification dataset ((MIT)-67). In our study, several existing, state-of-the art visual descriptors have been applied to the challenge of indoor versus outdoor scene categorization. The proposed SCGWD, Ohta ColorGIST wavelet descriptors, Ohta Color-GIST descriptor, and Enhanced Ohta color histogram descriptors have been compared with state-of-the-art visual descriptors, including CENTRIST-spatial pyramid, Color-GIST descriptors, Wavelet descriptors, Census Transform Histogram (CENTRIST), SIFT transformed with Sparse coding-based SPM (SIFTScSPM), SIFT with Locality-Constrained Linear Coding (SIFT-LLC), SIFT with SPM (SIFT-SPM), HOG with SPM (HOG-SPM), Speeded Up Robust Features with SPM (SURF-SPM), and Enhanced-GIST descriptors. The major contribution from the study has been integration of the proposed Ohta Color-GIST wavelet descriptors with CENTRIST (spatial pyramid) descriptors, to categorize indoor versus outdoor scene images for MAV navigation in GPS-denied indoor and outdoor environments.

\section{Enhanced Ohta color histogram descriptors (proposed visual descriptors)}

Ohta color space [32] is a linear transformation of RGB color space, in which color channels are computed for the indoor and outdoor image as follows:

$$
\begin{aligned}
& \mathrm{I} 1=\mathrm{R}+\mathrm{G}+\mathrm{B} \\
& \mathrm{I} 2=\mathrm{R}-\mathrm{B} \\
& \mathrm{I} 3=\mathrm{R}-2 \mathrm{G}+\mathrm{B}
\end{aligned}
$$

For each color channel, histogram features are extracted, using 32, 64, 128, and 256 bins, respectively, and then these features are concatenated, to form the Enhanced Ohta color histogram descriptors (480 dimensional descriptor).

Ohta color-GIST descriptors and Ohta color-GIST wavelet descriptors (proposed visual descriptors)

Ohta color histogram descriptors and Color-GIST descriptors are combined to produce Ohta Color-GIST descriptors (3552-dimensional descriptor $(3072+480))$. For each indoor and outdoor image, the Enhanced Ohta color histogram descriptors, Color-GIST descriptors, and wavelet descriptors are combined, to produce the proposed Ohta Color-GIST wavelet descriptors $(480+3072+22=3574$ - dimensional descriptor). 


\section{SCGWDs (proposed visual descriptor)}

SCGWDs are a new visual descriptor, created for the indoor versus outdoor scene classification task by combining Ohta Color-GIST wavelet descriptors with CENTRIST (spatial pyramid representation) descriptors. In this method, Enhanced Ohta color histogram descriptors were initially extracted, using 32, 64, 128, and 256 histogram bins. Then, Color-GIST descriptors were extracted from the image frame red, green, and blue channels, by applying 64 Gabor filters, at four scales and sixteen orientations, to obtain 64 feature maps. We then divided the 64 feature maps into $4 \times$ 4 grids and averaged the Gabor-filtered outputs to obtain the Color-GIST descriptors. In the next stage, wavelet descriptors were extracted by computing 2-D wavelet decomposition at level 3 on the "L" channel of the Lab color space image. The energy coefficients from the Laplacian-filtered approximation coefficients, and the horizontal, vertical, and diagonal detail coefficients were then combined, to produce wavelet descriptors. After this, CENTRIST (spatial pyramid) descriptors were extracted by dividing the image into 31 blocks and concatenating the histogram of the Census Transformed values into 31 image blocks, to produce spatial pyramid representations of the CENTRIST (spatial pyramid) descriptors. Finally, Enhanced Ohta color histogram descriptors, Color-GIST descriptors, wavelet descriptors, and CENTRIST (spatial pyramid) descriptors were combined, to produce the proposed SCGWDs.

\section{Implementation of indoor versus outdoor scene visual descriptors}

In this section, we have discussed using state-of-the-art visual descriptors and the proposed Ohta color-GIST descriptors, Ohta color-GIST wavelet descriptors, and SCGWDs for indoor versus outdoor scene classification.

To extract the Enhanced-GIST descriptor, the input grayscale image was divided into 16 regions, and 32 Gabor filters were applied, at 4 scales and 8 orientations, to obtain 32 GIST features. The 32 Gabor-filtered output responses within 16 regions were then averaged, to produce a 512-dimensional $(16 \times 32)$ GIST descriptor. Finally, the extracted GIST descriptors (512-dimensional) were combined with HODMG features (also 512-dimensional), to produce a 1024-dimensional, Enhanced-GIST descriptor.

Three different feature vector encoding methodsSPM, LLC and ScSPM-were used to encode the SIFTbased descriptor. To implement the SIFT-ScSPM, SIFTLLC, and SIFT-SPM descriptors, dense SIFT features were extracted from overlapping $16 \times 16$ patches, on dense grids, established every 8 pixels.

SIFT-ScSPM descriptors were extracted by applying sparse coding to the dense SIFT features, and then a linear SPM kernel, based on this sparse coding, was obtained by applying spatial max pooling. For SIFT-ScSPM use in our study, the sparse constraint parameter value was set at 0.15 , and a trained dictionary of 1024 words was used. This 1024-word, feature dictionary was obtained by applying the K-means clustering algorithm to local features. Finally, using the ScSPM algorithm, global features were produced from the dense SIFT descriptors. The feature vector encoding capabilities of the SIFTScSPM, SIFT-LLC, and SIFT-SPM descriptors were evaluated and compared-with the results shown in Table 1.

SIFT-SPM descriptors were extracted by applying the SPM algorithm from dense SIFT descriptors to global descriptors from the indoor and outdoor image. Three pyramid layers in the SPM scheme, and a codebook size of 400, were used here to extract the SIFT-SPM descriptors. A visual vocabulary of 400 visual words was obtained from the training set, by applying $\mathrm{K}$-means clustering to a random subset of patches, and then local features were quantized, using the trained visual words. Finally, SIFTSPM descriptors with 8400 bins were obtained, by concatenating the quantized SIFT feature histograms obtained from the indoor and outdoor image.

SIFT-LLC descriptors were extracted from the image by applying the LLC algorithm to dense SIFT features. A 1024-entry codebook, trained using the K-means clustering technique, was used to obtain the LLC codes from indoor and outdoor images. In the LLC feature extraction stage in our study, the K-nearest neighbor value was set to 5. Finally, max pooling was applied to the LLC codes of the dense SIFT descriptors, to produce SIFT-LLC descriptors.

HOG-SPM descriptors were extracted from overlapping $16 \times 16$ patches, by extracting local features from a dense grid which had a step size of 8 pixels. Firstly, image gradients were computed along the horizontal and vertical directions of the grayscale indoor and outdoor images, and then histograms were computed for multiple orientations, to produce HOG features. Global features were obtained by applying the SPM algorithm to the HOG descriptors. In HOG-SPM for this study, 3 pyramid layers (4200 dimension) and a 200-word codebook were used.

SURF-SPM descriptors were extracted by detecting SURF interest points (100 interest points) in $256 \times 256$ pixel resolution, grayscale images. Global features (SURFSPM) were obtained using the SPM algorithm, and in SURF-SPM, as used here, 3 pyramid layers (8400-dimension) and a codebook size of 400 and were used.

CENTRIST descriptors were extracted by comparing the center pixel to the intensity values of its $3 \times 3$ pixel neighborhood. Bit "0" was assigned to the neighboring pixel if the center pixel value was less than the neighboring pixels, otherwise bit "1" was assigned. The CENTRIST descriptors extracted from indoor and outdoor images were 256-dimensional descriptors. Spatial representation of CENTRIST descriptors was extracted by dividing the 
Table 1 Scene categorization performance on the Indian institute of technology madras-scene classification image database two dataset

\begin{tabular}{|c|c|c|c|c|c|c|}
\hline No & Algorithms & $C R$ & Precision & Recall & F-measure & AUC \\
\hline 1 & CENTRIST-spatial pyramid (RBF) & $92.93 \%$ & $92.93 \%$ & $92.91 \%$ & $92.92 \%$ & $92.92 \%$ \\
\hline 2 & CENTRIST-spatial pyramid (linear) & $92.53 \%$ & $92.53 \%$ & $92.54 \%$ & $92.53 \%$ & $92.54 \%$ \\
\hline 3 & Color-GIST descriptors (RBF) & $89.59 \%$ & $89.58 \%$ & $89.58 \%$ & $89.58 \%$ & $89.37 \%$ \\
\hline 4 & Color-GIST descriptors (linear) & $81.73 \%$ & $81.77 \%$ & $81.68 \%$ & $81.73 \%$ & $81.68 \%$ \\
\hline 5 & Wavelet descriptors (RBF) & $75.05 \%$ & $75.10 \%$ & $75.09 \%$ & $75.09 \%$ & $75.09 \%$ \\
\hline 6 & CENTRIST (linear) & $85.06 \%$ & $85.08 \%$ & $85.09 \%$ & $85.08 \%$ & $85.10 \%$ \\
\hline 7 & CENTRIST (RBF) & $86.24 \%$ & $86.27 \%$ & $86.28 \%$ & $86.28 \%$ & $86.28 \%$ \\
\hline 8 & SIFT-SCSPM (linear) & $92.92 \%$ & $92.92 \%$ & $92.94 \%$ & $92.93 \%$ & $92.94 \%$ \\
\hline 9 & SIFT-LLC (linear) & $92.53 \%$ & $92.52 \%$ & $92.54 \%$ & $92.53 \%$ & $92.55 \%$ \\
\hline 10 & SIFT-SPM (Chi-square) & $86.05 \%$ & $87.42 \%$ & $85.84 \%$ & $86.62 \%$ & $85.84 \%$ \\
\hline 11 & HOG-SPM (Chi-square) & $80.74 \%$ & $86.31 \%$ & $80.32 \%$ & $83.20 \%$ & $80.32 \%$ \\
\hline 12 & SURF- SPM (Chi-square) & $84.47 \%$ & $88.34 \%$ & $84.13 \%$ & $86.19 \%$ & $84.14 \%$ \\
\hline 13 & Enhanced-GIST (RBF) & $89.98 \%$ & $90.19 \%$ & $89.90 \%$ & $90.05 \%$ & $90 \%$ \\
\hline 14 & Enhanced-GIST (linear) & $84.28 \%$ & $84.40 \%$ & $84.21 \%$ & $84.21 \%$ & $84.22 \%$ \\
\hline 15 & Enhanced Ohta color histogram descriptors (RBF)-proposed & $76.03 \%$ & $77.04 \%$ & $76.23 \%$ & $76.63 \%$ & $76.23 \%$ \\
\hline 16 & Ohta Color-GIST descriptors (RBF)-proposed & $90.57 \%$ & $90.56 \%$ & $90.56 \%$ & $90.56 \%$ & $90.57 \%$ \\
\hline 17 & Ohta Color-GIST descriptors (linear)-proposed & $88.21 \%$ & $88.21 \%$ & $88.21 \%$ & $88.21 \%$ & $88.21 \%$ \\
\hline 18 & Ohta Color-GIST wavelet descriptors (linear)-proposed & $88.61 \%$ & $88.61 \%$ & $88.59 \%$ & $88.60 \%$ & $88.59 \%$ \\
\hline 19 & Ohta Color-GIST wavelet descriptors (RBF)-proposed & $90.57 \%$ & $90.56 \%$ & $90.56 \%$ & $90.56 \%$ & $90.57 \%$ \\
\hline 20 & spatial color-gist wavelet descriptors (linear) -proposed & $95.29 \%$ & $95.28 \%$ & $95.28 \%$ & $95.28 \%$ & $95.28 \%$ \\
\hline 21 & spatial color-gist wavelet descriptors (RBF)-proposed & $95.48 \%$ & $95.50 \%$ & $95.47 \%$ & $95.48 \%$ & $95.47 \%$ \\
\hline
\end{tabular}

AUC Area under the receiver operating characteristic curve, CR Classification rate, RBF Radial basis function kernel, SIFT-LLC SIFT with locality-constrained linear coding, SIFT-SCSPM SIFT with sparse coding based spatial pyramid matching, SIFT-SPM SIFT with spatial pyramid matching, SPM Spatial pyramid matching, HOG Histogram of oriented gradients, SURF Speeded up robust features, CENTRIST Census transform histogram

input image into 31 blocks, and the obtained histogram of Census Transformed values were concatenated into 31 blocks, to produce a 7936-dimensional descriptor.

To extract Wavelet descriptors, RGB input images were converted into CIELAB color space images. 2-D Wavelet decomposition, using biorthogonal wavelets (Bior 2.6), was applied, on the " $\mathrm{L}$ " channel of the Lab color space image. Wavelet energy coefficients-such as approximation coefficients (Laplacian-filtered coefficients), and horizontal, vertical, and diagonal detail coefficients-were extracted at level 3 and used as feature vectors. To extract the Enhanced Ohta color histogram descriptors, RGB images were converted into Ohta color space channels I1, I2, and I3, and Ohta color channel histograms were computed with 32, 64, 128, and 256 bins. The color histogram features were then concatenated, to produce Enhanced Ohta color histogram descriptors.

To extract Color-GIST descriptors, input RGB image red, green, and blue channels were each divided into 16 regions, and 64 Gabor filters were applied, at 4 scales and 16 orientations, to obtain 64 GIST feature maps. The 64 Gabor-filtered output responses within 16 regions were then averaged, to produce a 1024- dimensional $(16 \times 64)$ GIST descriptor. GIST descriptors extracted from each channel were then combined, to produce 3072-dimensional $(1024 \times 3)$ Color-GIST descriptors.

The extracted Enhanced Ohta color histogram descriptors and Color-GIST descriptors were combined, to produce the proposed Ohta Color-GIST descriptors-which were 3552-dimensional descriptors. The Enhanced Ohta color histogram descriptors, the Color-GIST descriptors, and the wavelet descriptors were then combined, to produce the proposed Ohta Color-GIST wavelet descriptors (3574-dimensional descriptors).

SCGWDs were then extracted for indoor and outdoor images by combining the Enhanced Ohta color histogram descriptors, the Color-GIST descriptors, the wavelet descriptors, and the CENTRIST (spatial pyramid) descriptors.

\section{Experimental evaluation and results}

This section presents an experimental evaluation of the proposed visual descriptor's performance, with respect to current, state-of the-art visual descriptors. All visual descriptors were evaluated using the IITM-SCID2 Dataset and Indoor-Outdoor Dataset, using five performance measures- 
$\mathrm{CR}$, precision, recall, F-measure, and area under the curve (AUC).

\section{Dataset for indoor versus outdoor scene recognition}

Scene classification experiments were conducted using the IITM-SCID2 and Indoor-Outdoor datasets. We used complex and confusing indoor and outdoor images, which required visual descriptors with strong discriminative ability to classify the indoor and outdoor scenes. Indoor scene classification experiments were conducted on the MIT-67 Dataset.

The IITM-SCID2 Dataset is a challenging benchmark dataset containing 902 images of indoor and outdoor scenes. From this dataset, 193 indoor images and 200 outdoor images were used in the training phase, to train the SVM classifier, while in the testing phase, 249 indoor images and 260 outdoor images were used, based on the trained scene recognition model. The Indoor-Outdoor Dataset consists of eight outdoor scene categories, including tall buildings, cityscapes, streets, highways, mountains, forests, open country, and coastal images, in a 4485-image dataset, and three indoor scene categories, including corridors, staircases, and rooms. Real time image frames were acquired from MAVs, with an image resolution of $1280 \times 720$ pixels. The dataset contained 1100 training images (300 indoor and 800 outdoor images), and 550 testing images (150 indoor and 400 outdoor images). The MIT-67 Dataset contains 15,620 images in 67 categories of complex indoor scenes. Here, 100 images or so from each of the 67 categories were chosen, so that around 6700 images were used for training and testing the classifier, using ten-fold cross validation, respectively.

\section{Performance measures and kernels used for indoor versus} outdoor scene recognition

In this paper, five different performance measures, including CR, precision, recall, f-measure, and AUC of the receiver operating characteristic (ROC) curve were used to assess indoor versus outdoor scene classification performance. In out testing, CR was defined as the percentage of correctly categorized test images, and four measures-true positives (TP), false positives (FP), false negatives (FN), and true negatives (TN) - could be computed, using the obtained confusion matrix.

Precision rate was expressed as shown in Eq. (3):

$$
P=\frac{T P}{T P+F N}
$$

where $T P$ and $F N$ represent the TP and FN, respectively.

Recall rate was expressed as shown in Eq. (4):

$$
R=\frac{T P}{T P+F P}
$$

where $T P$ and $F P$ represent the TP and FP, respectively.
The F-measure was defined as shown in Eq. (5):

$$
F=\frac{2 P R}{P+R}
$$

where $P$ and $R$ represent the precision rate and recall rate, respectively.

Area under the ROC curve, or AUC, was used as a performance measure to evaluate the indoor versus outdoor scene classification algorithm.

In this work, SVM with three different types of kernels-linear, RBF, and chi-squared-were used to classify the indoor and outdoor scenes.

The linear kernel for SVM classification could be computed as shown in Eq. (6):

$$
K\left(x_{i}, x_{j}\right)=x_{i} \cdot x_{j}
$$

where $x_{i}$ and $x_{j}$ represent two feature vectors.

The RBF kernel for SVM classification could then be computed as shown in Eq. (7):

$$
K\left(x_{i}, x_{j}\right)=\exp \left\{-\gamma\left\|x_{i}-x_{j}\right\|^{2}\right\}
$$

where $\gamma$ is a positive scalar, and $x_{i}$ and $x_{j}$ denote two feature vectors.

The Chi-square kernel could be then computed as shown in Eq. (8):

$$
k(x, y)=1-\sum_{i=1}^{N} \frac{2\left(x_{i}-y_{i}\right)^{2}}{\left(x_{i}+y_{i}\right)}
$$

where $x_{i}$ and $y_{i}$ represent two feature vectors. The Recognition rate, Precision $(\mathrm{P})$, Recall $(\mathrm{R}), \mathrm{F}$-measure $(\mathrm{F})$ and AUC were calculated, and the performance of the visual descriptors with SVM classifiers was evaluated using the IITM-SCID2 and Indoor-Outdoor datasets.

\section{Classification results using the IITM-SCID2, indoor- outdoor, and MIT-67 datasets}

Indoor versus outdoor scene classification results achieved on the IITM-SCID2 Dataset and Indoor-Outdoor Dataset using state-of-the-art visual descriptors and three proposed visual descriptors-Ohta color-GIST descriptors, Ohta color-GIST wavelet descriptors and SCGWD-have been listed in Tables 1 and 2. Scene classification experiments were conducted on a laptop computer equipped with an Intel i7-7500U CPU, operating at $2.70 \mathrm{GHz}$, and using 16 GB of RAM.

Experimental results indicated that, among the two datasets (the benchmark IITM-SCID2 Dataset and the Indoor-Outdoor Dataset), the IITM-SCID2 Dataset was the most difficult and challenging dataset from which to classify indoor and outdoor images.

The benchmark, eight-outdoor-scene data set (Outdoor images) included in the Indoor-Outdoor Dataset 
Table 2 Scene categorization performance on the Indoor Outdoor dataset

\begin{tabular}{|c|c|c|c|c|c|c|}
\hline No & Algorithms & $C R$ & Precision & Recall & F-measure & AUC \\
\hline 1 & CENTRIST-spatial pyramid (RBF) & $97.27 \%$ & $97.47 \%$ & $95.62 \%$ & $96.54 \%$ & $95.63 \%$ \\
\hline 2 & CENTRIST-spatial pyramid (linear) & $95.81 \%$ & $95.36 \%$ & $94 \%$ & $94.67 \%$ & $94 \%$ \\
\hline 3 & Color-GIST descriptors (RBF) & $89.63 \%$ & $92.89 \%$ & $81.41 \%$ & $86.77 \%$ & $81.42 \%$ \\
\hline 4 & Color-GIST descriptors (linear) & $83.27 \%$ & $78.84 \%$ & $81.83 \%$ & $80.31 \%$ & $81.83 \%$ \\
\hline 5 & Wavelet descriptors (RBF) & $74.54 \%$ & $69.11 \%$ & $71.45 \%$ & $70.26 \%$ & $71.46 \%$ \\
\hline 6 & CENTRIST (linear) & $93.63 \%$ & $92.88 \%$ & $90.83 \%$ & $91.84 \%$ & $90.83 \%$ \\
\hline 7 & CENTRIST (RBF) & $97.27 \%$ & $96.14 \%$ & $97.08 \%$ & $96.61 \%$ & $97.08 \%$ \\
\hline 8 & SIFT-SCSPM (linear) & $98.72 \%$ & $98.49 \%$ & $98.29 \%$ & $98.39 \%$ & $98.29 \%$ \\
\hline 9 & SIFT-LLC (linear) & $99.27 \%$ & $98.88 \%$ & $99.29 \%$ & $99.08 \%$ & $99.29 \%$ \\
\hline 10 & SIFT-SPM (Chi-square) & $57.81 \%$ & $51.75 \%$ & $52.04 \%$ & $51.89 \%$ & $52.04 \%$ \\
\hline 11 & HOG-SPM (Chi-square) & $71.81 \%$ & $51.79 \%$ & $50.20 \%$ & $50.98 \%$ & $50.21 \%$ \\
\hline 12 & SURF- SPM (Chi-square) & $73.27 \%$ & $86.56 \%$ & $51 \%$ & $64.18 \%$ & $51.00 \%$ \\
\hline 13 & Enhanced-GIST (RBF) & $97.81 \%$ & $96.73 \%$ & $97.87 \%$ & $97.30 \%$ & $97.88 \%$ \\
\hline 14 & Enhanced-GIST (linear) & $96.72 \%$ & $94.98 \%$ & $97.12 \%$ & $96.04 \%$ & $97.12 \%$ \\
\hline 15 & Enhanced Ohta color histogram descriptors (RBF)-proposed & $73.45 \%$ & $65.11 \%$ & $61.33 \%$ & $63.16 \%$ & $61.33 \%$ \\
\hline 16 & Ohta Color-GIST descriptors (RBF)-proposed & $98.73 \%$ & $98.29 \%$ & $98.50 \%$ & $98.39 \%$ & $98.50 \%$ \\
\hline 17 & Ohta Color-GIST descriptors (linear)-proposed & $96.72 \%$ & $94.86 \%$ & $97.33 \%$ & $96.08 \%$ & $97.33 \%$ \\
\hline 18 & Ohta Color-GIST wavelet descriptors (linear)-proposed & $97.27 \%$ & $95.70 \%$ & $97.70 \%$ & $96.69 \%$ & $97.71 \%$ \\
\hline 19 & Ohta Color-GIST wavelet descriptors (RBF)-proposed & $98.73 \%$ & $98.29 \%$ & $98.50 \%$ & $98.39 \%$ & $98.50 \%$ \\
\hline 20 & Spatial Color-gist wavelet descriptors (linear) -proposed & $99.45 \%$ & $99.21 \%$ & $99.42 \%$ & $99.31 \%$ & $99.42 \%$ \\
\hline 21 & spatial color-gist wavelet descriptors (RBF)-proposed & $99.82 \%$ & $99.67 \%$ & $99.87 \%$ & $99.77 \%$ & $99.88 \%$ \\
\hline
\end{tabular}

AUC Area under the receiver operating characteristic curve, CR Classification rate, RBF Radial basis function kernel, SIFT-LLC SIFT with locality-constrained linear coding, SIFT-SCSPM SIFT with sparse coding based spatial pyramid matching, SIFT-SPM SIFT with spatial pyramid matching, SPM Spatial pyramid matching, HOG Histogram of oriented gradients, SURF Speeded up robust features, CENTRIST Census transform histogram

was the easiest to classify, in comparison to the outdoor images in the IITM-SCID2 Dataset. The highest average CRs-95.48\% and $99.82 \%$ using the RBF kernel, and $95.29 \%$ and $99.45 \%$ using the linear kernelattained from the IITM-SCID2 Dataset and IndoorOutdoor Dataset were achieved by the SCGWDs. CR, Precision, Recall, F-measure, and area under the ROC (AUC) performance measures indicated that the SCGWD achieved results better than those of the state-of-the-art visual descriptors.

The proposed method could be used as a scene classifier for navigating MAVs in indoor and outdoor environments. The results inferred that the combination of color features (Enhanced Ohta color histogram descriptors and color-GIST descriptors) and texture features [Wavelet descriptors and CENTRIST (spatial pyramid representation)] was very effective in classifying indoor and outdoor scenes.

Our study was a comparative study between several state-of-the-art visual descriptors and the proposed Ohta color-GIST descriptors, Ohta color-GIST wavelet descriptors and SCGWD, from both methodological and experimental perspectives, for indoor versus outdoor scene and indoor scene classification tasks. Indoor scene SCGWD classification results achieved using the MIT-67 dataset have been listed in Table 3, and CRs of

Table 3 Computational cost calculation

\begin{tabular}{|c|c|c|c|c|c|c|c|}
\hline \multirow[t]{2}{*}{ Type of feature } & \multirow{2}{*}{$\begin{array}{l}\text { SVM } \\
\text { classifier } \\
\text { method }\end{array}$} & \multicolumn{2}{|c|}{ IITM-SCID2 Dataset } & \multicolumn{2}{|c|}{ Indoor-Outdoor Dataset } & \multicolumn{2}{|c|}{$\begin{array}{l}\text { MIT-67 indoor scene classification } \\
\text { Dataset }\end{array}$} \\
\hline & & $\begin{array}{l}\text { Recognition } \\
\text { rate }\end{array}$ & $\begin{array}{l}\text { Average time elapsed, } \\
\text { in seconds per frame }\end{array}$ & $\begin{array}{l}\text { Recognition } \\
\text { rate }\end{array}$ & $\begin{array}{l}\text { Average time elapsed, } \\
\text { in seconds per frame }\end{array}$ & $\begin{array}{l}\text { Recognition } \\
\text { rate }\end{array}$ & $\begin{array}{l}\text { Average time elapsed, } \\
\text { in seconds per frame }\end{array}$ \\
\hline \multirow[t]{2}{*}{$\begin{array}{l}\text { Spatial color gist } \\
\text { wavelet descriptors }\end{array}$} & $\begin{array}{l}\text { linear } \\
\text { kernel }\end{array}$ & $95.29 \%$ & 2.58 & $99.45 \%$ & 2.80 & $4.92 \%$ & 1.74 \\
\hline & $\begin{array}{l}\text { RBF } \\
\text { kernel }\end{array}$ & $95.48 \%$ & 2.60 & $99.82 \%$ & 2.89 & $2.08 \%$ & 2.12 \\
\hline
\end{tabular}




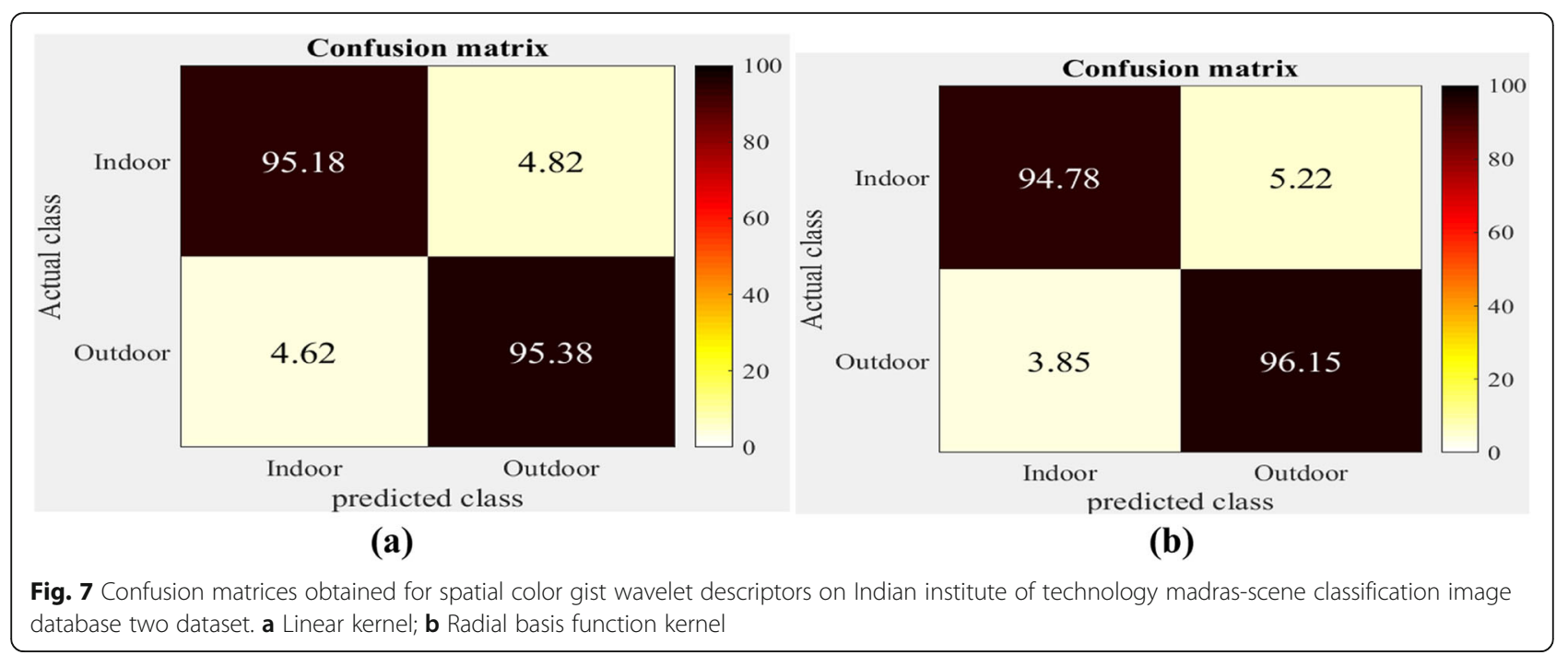

$2.08 \%$ and $4.92 \%$ were obtained using the RBF and linear kernels.

These experimental results indicated that SCGWDs performed better for indoor versus outdoor scene categorization as opposed to indoor scene categorization. In fact, the SCGWDs were proposed mainly for indoor versus outdoor scene categorization, but descriptor effectiveness was also tested, and was found to be unsuitable for the indoor scene classification task, as the spatial layout, color, and texture information extracted from indoor scenes using the SCGWD was insufficient for recognition of indoor scenes.

Compared to other visual descriptors, SCGWD had the highest CR on the IITM-SCID2 Dataset and IndoorOutdoor Dataset, as shown in Tables 1 and 2.
The confusion matrices obtained when applying the SCGWD to the IITM-SCID2 and Indoor-Outdoor datasets are shown in Figs. 7 and 8, respectively. The rows and columns in the confusion matrices correspond to actual and predicted classes, respectively, while the diagonal values show the average CR for each indoor and outdoor image category.

Computation times taken, per frame, have been documented in Table 3.

\section{Conclusions}

Indoor versus outdoor scene classification is difficult, due to intra-class variability, inter-class similarity in the case of indoor scenes, and the variability of outdoor

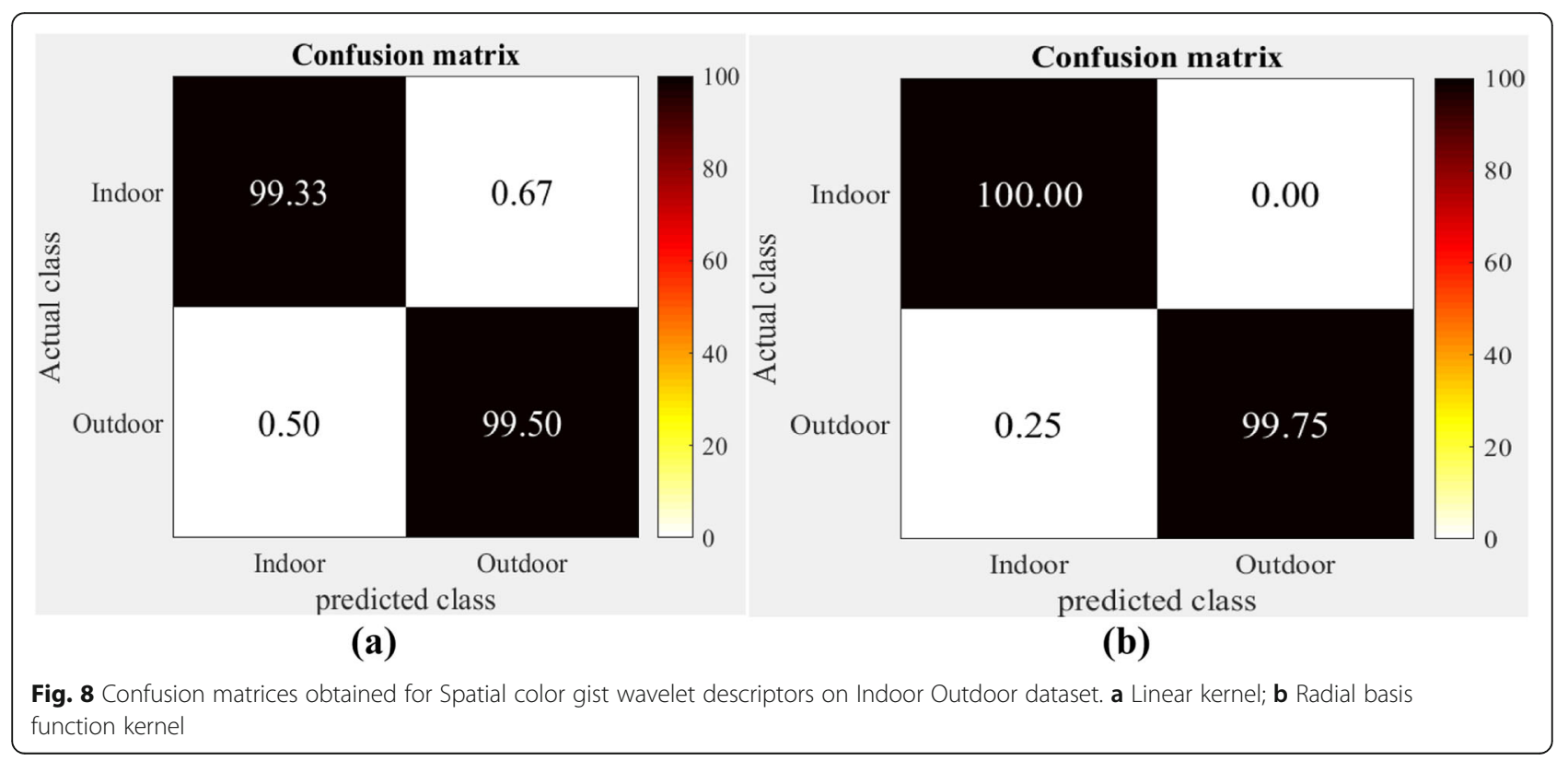


scene content caused by different weather conditions and the nature of the objects involved in outdoor scenes. In this paper, a new visual descriptor, called the SCGWD and based on the combination of proposed Ohta color-GIST wavelet descriptors and CENTRIST (spatial pyramid representation) descriptors for Indoor-Outdoor scene classification task, has been described. The proposed new visual descriptor consists of color, texture, and spatial information content of the scene. When faced with the IITM-SCID2 dataset and Indoor-Outdoor Dataset, SCGWDs produced recognition rates of $95.48 \%$ and $99.82 \%$, using SVM with an RBF kernel, and $95.29 \%$ and $99.45 \%$ using SVM with a linear kernel. Furthermore, using SCGWDs for scene classification, higher $\mathrm{CR}$, precision, recall, and area under the ROC curve values were obtained, with respect to other, state-of-the-art visual descriptors.

In contrast, CRs of only $2.08 \%$ and $4.92 \%$ were obtained using RBF and linear kernels with the MIT-67 dataset, which showed that the SCGWD was unsuitable for use in the categorization of complex, 67 indoor scene image categories. Overall, however, we were able to conclude that the proposed scene recognition algorithm could be used as a scene classifier (Indoor versus Outdoor) for navigating MAVs.

\begin{abstract}
Abbreviations
2-D: 2 Dimensional; AR: Augmented reality; AUC: Area under the receiver operating characteristic curve; CENTRIST: Census transform histogram; CR: Classification rate; CT: Census transform; FN: False negatives; FP: False positives; GPS: Global positioning systems; HOG: Histogram of oriented gradients; HOG-SPM: HOG with spatial pyramid matching; IITM-SCID2: Indian institute of technology madras-scene classification image database two; LLC: Locality-constrained linear coding; MAV: Micro aerial vehicle; MIT: Massachusetts Institute of Technology; RBF: Radial basis function kernel; ROC: Receiver operating characteristic; SCSPM: Sparse coding based spatial pyramid matching; SIFT: Scale-invariant feature transform; SIFT-LLC: SIFT with locality-constrained linear coding; SIFT-SCSPM: SIFT with sparse coding based spatial pyramid matching; SIFT-SPM: SIFT with spatial pyramid matching; SPM: Spatial pyramid matching; SUN: Scene understanding; SURF: Speeded up robust features; SURF-SPM: SURF with spatial pyramid matching; SVM: Support vector machine; TN: True negatives; TP: True positives; VQ: Vector quantization
\end{abstract}

\section{Acknowledgements}

Not applicable

\section{Authors' contributions}

Both authors read and approved the final manuscript.

\section{Funding}

Not applicable.

\section{Availability of data and materials}

The datasets used and/or analyzed for indoor versus outdoor scene categorization and indoor scene categorization are available from the corresponding author on reasonable request.

\section{Competing interests}

The authors declare that they have no competing interests.
Received: 1 August 2019 Accepted: 30 October 2019

Published online: 26 November 2019

\section{References}

1. Gupta L, Pathangay V, Patra A, Dyana A, Das S (2006) Indoor versus outdoor scene classification using probabilistic neural network. EURASIP J Adv Signal Process 2007:094298. https://doi.org/10.1155/2007/94298

2. Payne A, Singh S (2005) Indoor vs. outdoor scene classification in digital photographs. Pattern Recogn 38(10):1533-1545. https://doi.org/10.1016/j. patcog.2004.12.014

3. Lai YK, Rosin PL (2014) Efficient circular thresholding. IEEE Trans Image Process 23(3):992-1001. https://doi.org/10.1109/TIP.2013.2297014

4. Szummer M, Picard RW (1998) Indoor-outdoor image classification. In: Proceedings of 1998 IEEE international workshop on content-based access of image and video database. IEEE, Bombay. https://doi.org/10.1109/CAIVD. 1998.646032

5. Quattoni A, Torralba A (2009) Recognizing indoor scenes. In: Proceedings of 2009 IEEE conference on computer vision and pattern recognition. IEEE, Miami. https://doi.org/10.1109/CVPR.2009.5206537

6. Xie LX, Wang JD, Guo BN, Zhang B, Tian Q (2014) Orientational pyramid matching for recognizing indoor scenes. In: Proceedings of 2014 IEEE conference on computer vision and pattern recognition. IEEE, Columbus. https://doi.org/10.1109/CVPR.2014.477

7. Cakir F, Güdükbay U, Ulusoy Ö (2011) Nearest-neighbor based metric functions for indoor scene recognition. Comput Vis Image Underst 115(11): 1483-1492. https://doi.org/10.1016/j.cviu.2011.07.007

8. Qin JZ, Yung NHC (2010) Scene categorization via contextual visual words. Pattern Recogn 43(5):1874-1888. https://doi.org/10.1016/j.patcog. 2009.11.009

9. Xiao JX, Hays J, Ehinger KA, Oliva A, Torralba A (2010) SUN database: largescale scene recognition from abbey to zoo. In: Proceedings of 2010 IEEE computer society conference on computer vision and pattern recognition, IEEE, San Francisco. https://doi.org/10.1109/CVPR.2010.5539970

10. Kawewong A, Pimpup R, Hasegawa O (2013) Incremental learning framework for indoor scene recognition. In: Proceedings of the TwentySeventh AAAl conference on artificial intelligence. AAAI Press, Bellevue, Washington

11. Khan SH, Hayat M, Bennamoun M, Togneri R, Sohel FA (2016) A discriminative representation of convolutional features for indoor scene recognition. IEEE Trans Image Process 25(7):3372-3383. https://doi.org/10. 1109/TIP.2016.2567076

12. Meng $X L$, Wang $Z Z$, Wu LZ (2012) Building global image features for scene recognition. Pattern Recogn 45(1):373-380. https://doi.org/10.1016/j.patcog. 2011.06.012

13. Liu SY, Xe D, Feng SH (2011) Region contextual visual words for scene categorization. Expert Syst Appl 38(9):11591-11597. https://doi.org/10.1016/j. eswa.2011.03.037

14. Liao B, Xu JG, LV JT, Zhou SL (2015) An image retrieval method for binary images based on DBN and softmax classifier. IETE Tech Rev 32(4):294-303. https://doi.org/10.1080/02564602.2015.1015631

15. Mohan BK (2000) Classification of remotely sensed images using artificial neural networks. IETE J Res 46(5):401-410. https://doi.org/10.1080/03772063. 2000.11416184

16. Elfiky NM, Khan FS, Van De Weijer J, Gonzàlez J (2012) Discriminative compact pyramids for object and scene recognition. Pattern Recogn 45(4): 1627-1636. https://doi.org/10.1016/j.patcog.2011.09.020

17. Yang JC, Yu K, Gong YH, Huang T (2009) Linear spatial pyramid matching using sparse coding for image classification. In: Proceedings of 2009 IEEE conference on computer vision and pattern recognition. IEEE, Miami

18. Lazebnik S, Schmid C, Ponce J (2006) Beyond bags of features: spatial pyramid matching for recognizing natural scene categories. In: Proceedings of 2006 IEEE computer society conference on computer vision and pattern recognition. IEEE, New York

19. Wei X, Phung SL, Bouzerdoum A (2016) Visual descriptors for scene categorization: experimental evaluation. Artif Intell Rev 45(3):333-368. https://doi.org/10.1007/s10462-015-9448-4

20. Ghomsheh AN, Talebpour A (2012) A new method for indoor-outdoor image classification using color correlated temperature. Int J Image Process 6(3):167-181 
21. Anbarasu B, Anitha G (2019) Indoor scene recognition for micro aerial vehicles navigation using enhanced SIFT-ScSPM descriptors. J Navigation. https://doi.org/10.1017/S0373463319000420

22. Tong ZH, Shi DX, Yang BZ, Wei J (2017) A review of indoor-outdoor scene classification. In: Proceedings of the Second international conference on control, automation and artificial intelligence. CAAl, Sanya. https://doi.org/ 10.2991/caai-17.2017.106

23. Lowe DG (2004) Distinctive image features from scale-invariant keypoints. Int J Comput Vis 60(2):91-110. https://doi.org/10.1023/B:VISI.0000029664. 99615.94

24. Bay H, Ess A, Tuytelaars T, Van Gool L (2008) Speeded-up robust features (SURF). Comput Vis Image Underst 110(3):346-359. https://doi.org/10.1016/j. cviu.2007.09.014

25. Dalal N, Triggs B (2005) Histograms of oriented gradients for human detection. In: Proceedings of 2005 IEEE computer society conference on computer vision and pattern recognition. IEEE, San Diego. https://doi.org/10. 1109/CVPR.2005.177

26. Wu JX, Rehg JM (2011) CENTRIST: a visual descriptor for scene categorization. IEEE Trans Pattern Anal Mach Intell 33(8):1489-1501. https:// doi.org/10.1109/TPAMI.2010.224

27. Anbarasu B, Anitha G (2018) Indoor scene recognition for micro aerial vehicles navigation using enhanced-GIST descriptors. Defense Sci J 68(2): 129-137. https://doi.org/10.14429/dsj.68.10504

28. Haralick RM, Sternberg SR, Zhuang XH (1987) Image analysis using mathematical morphology. IEEE Trans Pattern Anal Mach Intell PAMI-9(4): 532-550. https://doi.org/10.1109/TPAMl.1987.4767941

29. Wang JJ, Yang JC, Yu K, Lv FJ, Huang T, Gong YH (2010) Localityconstrained linear coding for image classification. In: Proceedings of 2010 IEEE computer society conference on computer vision and pattern recognition. IEEE, San Francisco. https://doi.org/10.1109/CVPR.2010.5540018

30. Prakash O, Khare M, Srivastava RK, Khare A (2013) Multiclass image classification using multiscale biorthogonal wavelet transform. In: Proceedings of 2013 IEEE Second international conference on image information processing. IEEE, Shimla. https://doi.org/10.1109/ICIIP.2013. 6707569

31. Oliva A, Torralba A (2006) Building the gist of a scene: the role of global image features in recognition. Prog Brain Res 155:23-36. https://doi.org/10. 1016/50079-6123(06)55002-2

32. Ohta YI, Kanade T, Sakai T (1980) Color information for region segmentation. Comput Graphics Image Process 13(3):222-241. https://doi.org/10.1016/ 0146-664X(80)90047-7

\section{Publisher's Note}

Springer Nature remains neutral with regard to jurisdictional claims in published maps and institutional affiliations.

\section{Submit your manuscript to a SpringerOpen ${ }^{\circ}$ journal and benefit from:}

- Convenient online submission

- Rigorous peer review

- Open access: articles freely available online

- High visibility within the field

- Retaining the copyright to your article

Submit your next manuscript at $\boldsymbol{\nabla}$ springeropen.com 